\title{
Compact five-band antenna array with vertical polarization
}

\author{
Vladimir Taranenko* \\ Guandong Sigtenna Communication Technology Co., Ltd., Jin da Automobile logistic city, No. 4, \\ Yanwo village, Shipai Town, Dongguan, China
}

\begin{abstract}
A vertical polarized antenna array operating in frequency bands $423-443,840-930,1525-1625,2400-2500$ and $5725-5850 \mathrm{MHz}$ is presented. The radiators are made in a shape of stacked patches excited by metal strips located under the radiating patches. The radiating patch of the lowest frequency band $423-443 \mathrm{MHz}$ is made in the form of a printed circuit board and acts as a reflector for radiators operating at other frequency bands. Such two-storey structure provides small dimensions of the antenna array. The results of simulation are compared with the measured radiation patterns and $\mathrm{S}$ parametes of the manufactured antenna array.
\end{abstract}

\section{Introduction}

Modern mobile communication systems often use multiple frequency bands to increase the amount of transmitted information and the reliability of communication channels. Such systems require antennas operating in several frequency bands [1,2]. Dimensions of multiband antennas must be as small as possible therefore radiators operating in different frequency bands are located close to each other and affect each other. The design goal is to provide the specified radiation patterns and matching all radiators with the minimum dimensions of the antenna. The present work investigates the possibility of reducing the dimensions of the vertical polarized antenna array operating in five frequency bands: 423-443, 840-930, 1525 $1625,2400-2500$ and $5725-5850 \mathrm{MHz}$. The antenna array having specified dimensions of $401 \mathrm{~mm} \times 355 \mathrm{~mm} \times 86 \mathrm{~mm}$ must provide beamwidth at horizontal plane about 60 degrees at $-3 \mathrm{~dB}$ and maximum gain for all five frequency bands.

\section{Antenna array structure design}

Since the specified frequency bands are quite narrow, to ensure the specified requirements, vertical polarization and width of the antenna radiation patterns, it was decided to use simple radiators in a shape of stacked patches excited by metal strips located under the radiating patches. Due to the large size of the radiator operating at the lowest frequency band, it was decided to use it as a reflector for radiators operating at other frequency bands. This two-story antenna structure, shown in Figure 1, allowed to dispose the all radiators within the specified dimensions of $401 \mathrm{~mm} \times 355 \mathrm{~mm} \times 86 \mathrm{~mm}$.

\footnotetext{
*Corresponding author: vladimirtaranenko.v@yandex.ru
} 
The radiating patch operating at the lowest frequency band is made in the form of a printed circuit board (PCB) made of FR-4 material having a thickness of $1.5 \mathrm{~mm}$ and dielectric permittivity of 4.5 . Radiators of other frequency band and the power dividers feeding them are disposed on the top surface of PCB mounted on the metal reflector. The horizontal edges of the metal reflector are bent upwards to reduce back radiation. The vertical edges of the reflector are bent down and under the reflector to narrow the horizontal beam width. The reflector has longitudinal rectangular cutouts of a certain size. Such periodic structure reduces shunt effect of the reflector on the radiating patch therefore it is possible to dispose one at a small distance from the reflector, thereby narrow-

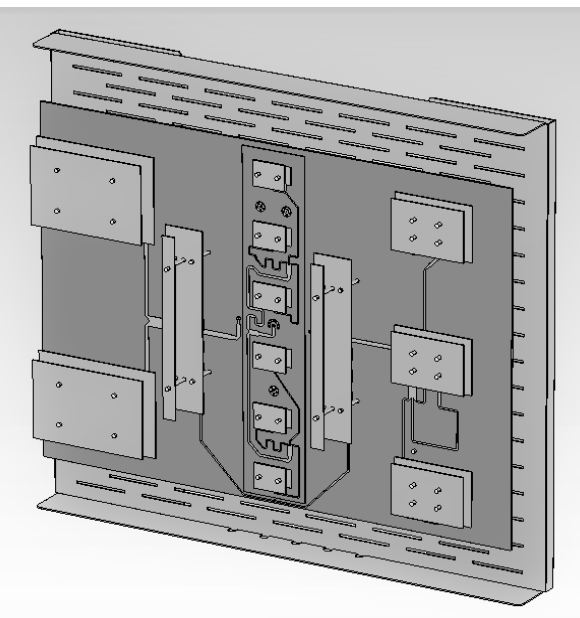

Fig. 1. Appearance of the antenna array. ing its beam width and reducing a back lobe, while ensuring specified VSWR.

The radiators of the second, third and fourth bands have similar structures to each other. They are made in a shape of rectangular aluminum patches mounted on plastic spacers and excited by metal strips located under the radiating patches. The upper rectangular aluminum patches improve VSWR of the radiators. The antenna array of the second band is made of two horizontally spaced and parallel connected identical radiators. Each radiator consists of two narrow patches. Such design frees up space between the radiators of the second band for disposition of the fifth band antenna array. The antenna array of the third band located at the left edge of the PCB, is made of two vertically spaced identical radiators feeded by a microstrip power divider formed on the top surface of PCB. The antenna of the fourth band, located at the right edge of PCB, is made of three vertically spaced identical radiators feeded by a 3-way microstrip power divider.

The antenna array of the fifth band are formed on the second PCB having thickness of $0.76 \mathrm{~mm}$ and dielectric permittivity of 3.3, manufactured of high quality dielectric RO4533, providing small dissipative losses in microstrip lines. The second PCB is disposed in the center of first PCB. The antenna array contains six radiators in a shape of microstrip patches formed on the top surface of the second PCB together with the feeding power dividers. The rectangular aluminum patches are mounted above microstrip patches on plastic spacers to improve VSWR of the radiators.

Power dividers feeding radiators of the second, third and fours bands are formed on the top surface of the first PCB and its inputs are disposed at horizontal axis of PCB where intensity of electric field is minimal. Coaxial cables connected to the inputs of all power dividers pass from the bottom surface of $\mathrm{PCB}$ to the middle of the reflector where intensity of electric field is minimal and are disposed along the reflector to the horizontal side wall of the reflector where the coaxial connectors are mounted.

Disposition and feeding of radiators of the antenna array in the proposed way allowed to use the specified dimensions efficiently and meet the requirements for the gain, the width of the radiation patterns in the horizontal plane and VSWR for all frequency bands. The design goal was achieved by using two-storey antenna structure, the main reflector of a special shape and radiators containing additional passive patches. 


\section{Simulated and measured results}

The electromagnetic analysis of this structure is made by CAD. The second, third and fourth bands of a prototype antenna are measured. The results of simulation are compared with the measured results.

The simulated and measured radiation pattern at central frequency $(\mathrm{CF})$ for the second, third and fourth range is shown in Figs. 2 - 4.
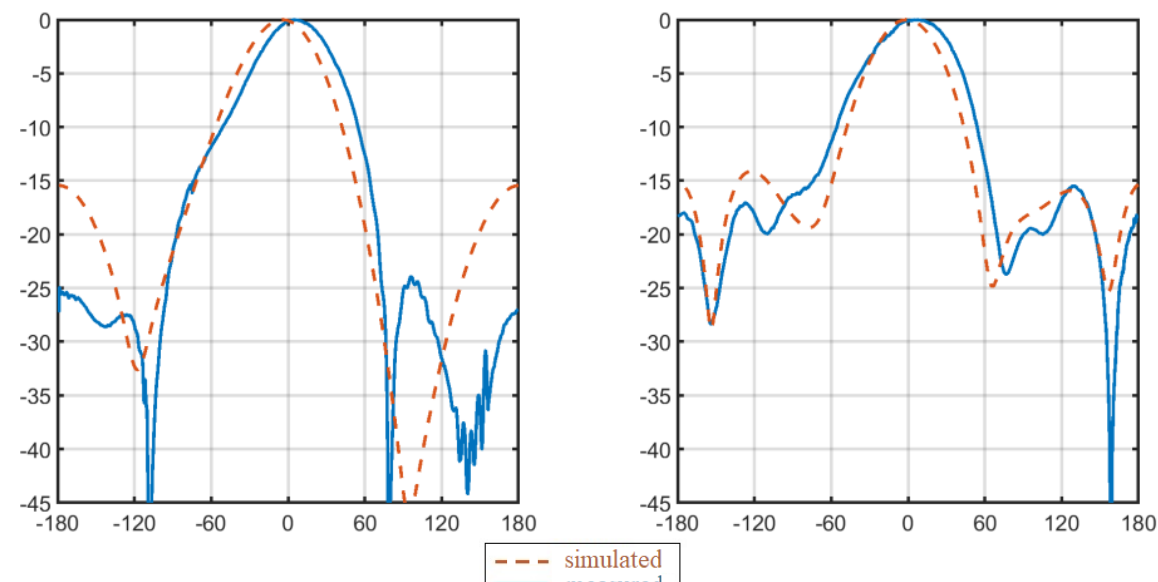

(a)

(b)

Fig. 2. Simulated and measured patterns at $\mathrm{CF}=885 \mathrm{MHz}$

a) horizontal plane b) vertical plane
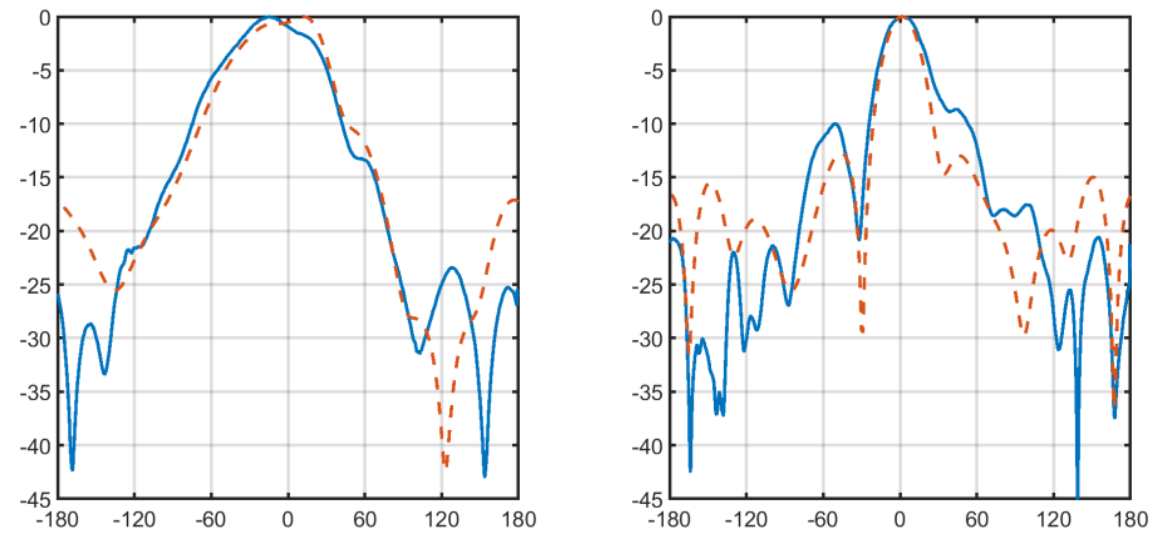

(a)

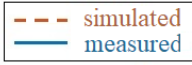

(b)

Fig. 3. Simulated and measured patterns at $\mathrm{CF}=1575 \mathrm{MHz}$

a) horizontal plane b) vertical plane 

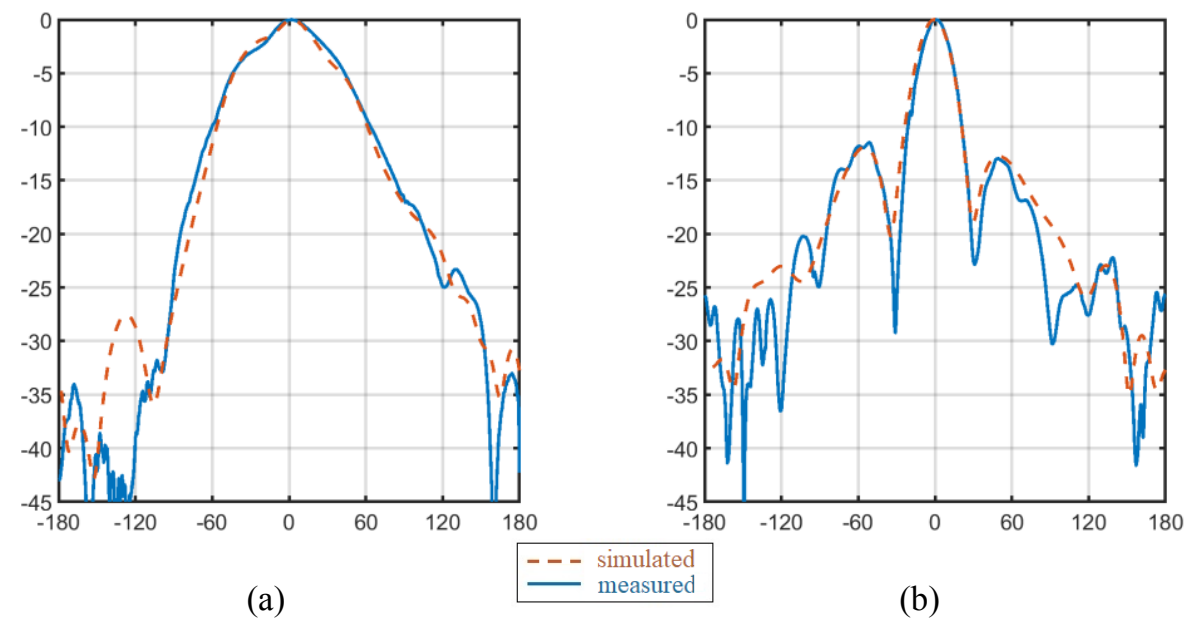

(a)

$\begin{array}{ll}--- & \text { simulated } \\ \text { measured }\end{array}$

(b)

Fig. 4. Simulated and measured patterns at $\mathrm{CF}=2450 \mathrm{MHz}$

a) horizontal plane b) vertical plane
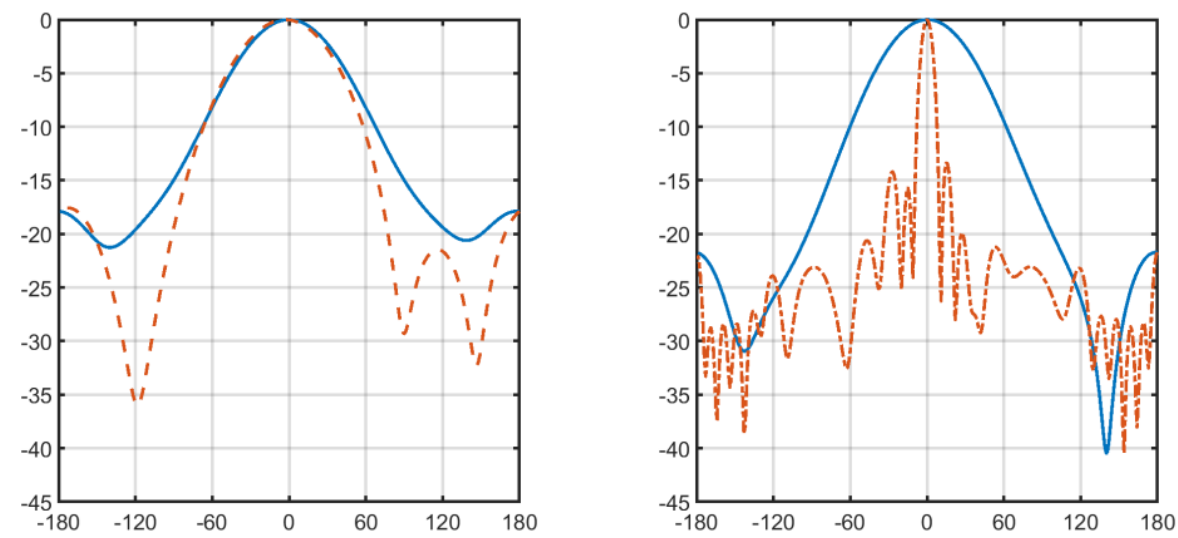

Fig. 5. Simulated radiation pattern at $\mathrm{CF}=433 \mathrm{MHz}$ in vertical and horizontal planes

Fig. 6. Simulated radiation pattern at $\mathrm{CF}=5787 \mathrm{MHz}$ in vertical and horizontal planes

Shown above patterns are asymmetric ones. Reflections from disposed nearby radiators operating at other frequency bands causes asymmetry at horizontal plane. Not symmetric feeding of radiating patched causes asymmetry at vertical plane. Such asymmetry could be decreased by feeding patches.

The radiation patterns of the antennas operating at 423-443 and 5725-5850 MHz was only simulated. Fig. 5 and Fig. 6 illustrate radiation patterns at $\mathrm{CF}=433 \mathrm{MHz}$ and $\mathrm{CF}=$ $5787 \mathrm{MHz}$ correspondingly in vertical and horizontal planes.

The radiation pattern shown in Fig. 6 is significantly distorted by reflections from disposed near antennas operating at other bands. Fig. 7 illustrates the simulated radiation pattern of this antenna array disposed separately from other antennas. In order to reduce distortions shown in Fig. 6 the second PCB forming the antenna array for the fifth frequency band was disposed at $20 \mathrm{~mm}$ distance above the first PCB. The improved simulated radiation pattern of the antenna array at the new position are shown in Fig. 8. 

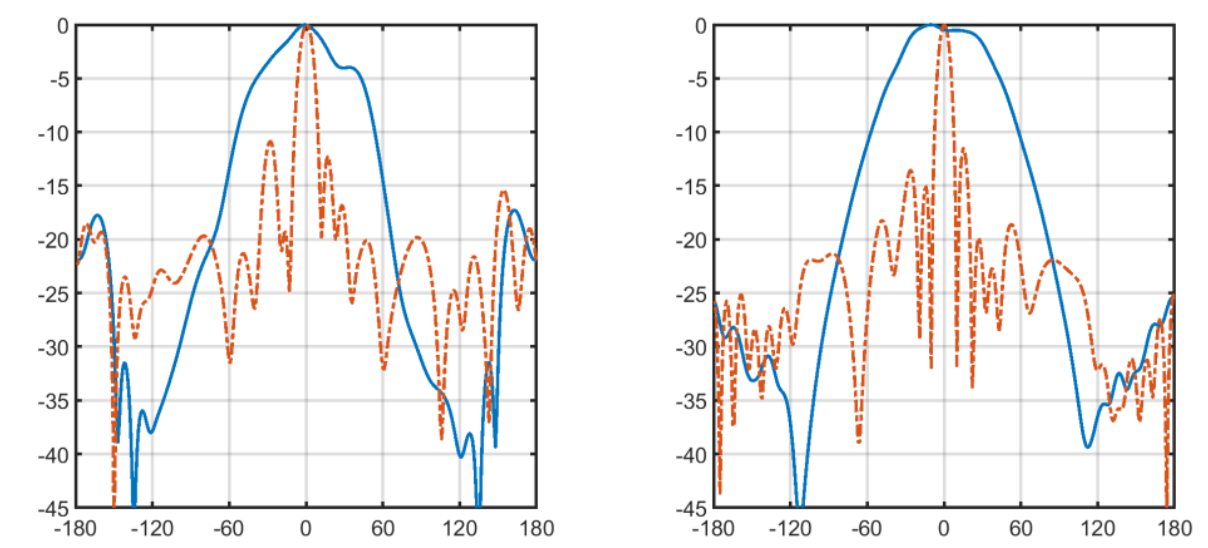

Fig. 7. Simulated radiation pattern ----- vertical horizontal of a single antenna at $\mathrm{CF}=5787 \mathrm{MHz}$ in vertical and horizontal planes.

Fig. 8. Simulated radiation pattern of the moved antenna at $\mathrm{CF}=5787$ MHzin vertical and horizontal planes.

The comparison of simulated and measured results is shown in Table 1.

Table 1. The simulated and measured results of the developed antenna array

\begin{tabular}{|c|c|c|c|c|c|}
\hline $\begin{array}{c}\text { Frequency band } \\
\text { [MHz] }\end{array}$ & $423-443$ & $840-930$ & $\begin{array}{c}1525- \\
1625\end{array}$ & $\begin{array}{c}2400- \\
2500\end{array}$ & $\begin{array}{c}5725- \\
5850\end{array}$ \\
\hline Simulated gain [dBi] & 8 & 10 & 11 & 13 & 16 \\
\hline $\begin{array}{c}\text { Simulated beamwidth } \\
\text { at the CF in horizon- } \\
\text { tal / vertical planes [ }\end{array}$ & $69.6 /$ & $\begin{array}{c}52.5 / \\
50.7\end{array}$ & $\begin{array}{c}66.3 / \\
27\end{array}$ & $\begin{array}{c}59.3 / \\
24.5\end{array}$ & $69 / 9$ \\
\hline $\begin{array}{c}\text { Measured beamwidth } \\
\text { at the CF in horizontal } \\
\text { / vertical [ }{ }^{\circ} \text { ] }\end{array}$ & $1.5 / 1.4$ & $1.2 / 1.5$ & $1.2 / 1.35$ & $1.4 / 1.2$ & $1.6 / 1.4$ \\
\hline $\begin{array}{c}\text { VSWR simulated / } \\
\text { measured }\end{array}$ & 62 & $\begin{array}{c}66 / \\
32.1\end{array}$ & $\begin{array}{c}61.3 / \\
22.2\end{array}$ & \\
\hline
\end{tabular}

\section{Conclusion}

In this paper the new compact design of the five-band antenna array providing linear polarization is presented. The specified radiation pattern beamwidths about $60^{\circ}$ at the level of -3 $\mathrm{dB}$, gain and VSWR are achieved. The next stage of this work is investigation of the possibility of reducing the mutual influence of neighboring radiators.

\section{References}

1. Seong-Ook Park, Viet-Anh Nguyen and Rao Shahid Aziz, Multi-band, dual polarization, dual antennas for beam reconfigurable antenna system for small cell base station , The 2014 International Workshop on Antenna Technology (2014)

2. Xin Sun, Fan Chun, Gang Zeng and Hong-chun Yang, A novel compact planar multiband antenna for GPS, WLAN and WIMAX applications, China-Japan Joint Microvave Conference (2011) 